\title{
SELENIUM AND COGNITIVE DECLINE IN THE ELDERLY - THE EVA STUDY
}

\author{
C. Berr, B. Balansard, J. Arnaud, A. M. Roussel, and A. Alpérovitch \\ INSERM U360, Hôpital de la Salpêtrière \\ 75651-Paris Cedex 13 and \\ Laboratoire de biologic du stress oxydant \\ Université de Grenoble I \\ 38700, La Tronche \\ France
}

The aim of this study was to determine whether systemic oxidative stress status is associated with cognitive decline in elderly community subjects. We studied vascular and cerebral aging in 1166 high cognitive functioning subjects aged 60-70 years volunteers in the EVA ("Etude du Vieillissement Artériel") cohort with a 4-year follow-up. Subjects completed a global cognitive test (MMSE) at baseline and during the 4-year follow-up examination. Blood samples were obtained at baseline to determine plasma levels of selenium. Associations between selenium levels and demographic (age, sex, socio-economic level), lifestyle (alcohol, tobacco), clinical and metabolic (lipids, glycemia) factors were examined. Risk of cognitive decline, defined as a loss of 3 points in MMSE score between baseline and the 4-year follow-up, was assessed by selenium level.

Mean level of selenium was equal to $1.10 \mu \mathrm{mol} / 1(\mathrm{sd}=0.2)$. Education and income levels were positively associated to selenium. There was also a positive association between selenium and cholesterol. Age, sex, alcohol and tobacco consumption were not significantly associated with selenium in this sample.

Subjects with low levels of selenium (defined as lower than $25^{\text {th }}$ percentile, $0.96 \mu \mathrm{mol} / \mathrm{l}$ ) have an increased risk of cognitive decline (Odds ratio $=1.58$ confidence interval 95\% (1.08-2.31)) after adjustment for various confounding (sex, age, depressive symptomatology, educational level, alcohol and tobacco consumption, BMI, cholesterol, triglycerides, and initial MMSE score). Analysis did not show a dose-effect relationship between selenium levels and risk of cognitive decline. This result suggest that antioxidant deficiencies may accelerate cognitive decline or be a risk factor for cognitive decline. 